\title{
Anvil cell gasket design for high pressure nuclear magnetic resonance experiments beyond $30 \mathrm{GPa}$
}

Thomas Meier, and Jürgen Haase

Citation: Review of Scientific Instruments 86, 123906 (2015); doi: 10.1063/1.4939057

View online: https://doi.org/10.1063/1.4939057

View Table of Contents: http://aip.scitation.org/toc/rsi/86/12

Published by the American Institute of Physics

\section{Articles you may be interested in}

Moissanite anvil cell design for giga-pascal nuclear magnetic resonance

Review of Scientific Instruments 85, 043903 (2014); 10.1063/1.4870798

High sensitivity nuclear magnetic resonance probe for anvil cell pressure experiments

Review of Scientific Instruments 80, 073905 (2009); 10.1063/1.3183504

Nuclear magnetic resonance in a diamond anvil cell at very high pressures

Review of Scientific Instruments 69, 479 (1998); 10.1063/1.1148686

Magnetic flux amplification by Lenz lenses

Review of Scientific Instruments 84, 085120 (2013); 10.1063/1.4819234

A cubic boron nitride gasket for diamond-anvil experiments

Review of Scientific Instruments 79, 053903 (2008); 10.1063/1.2917409

Theory of the gasket in diamond anvil high-pressure cells

Review of Scientific Instruments 60, 3789 (1989); 10.1063/1.1140442

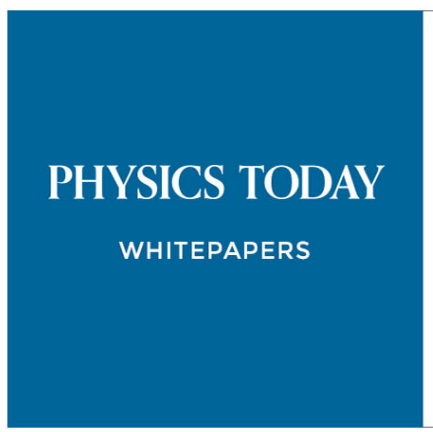

MANAGER'S GUIDE

Accelerate R\&D with

Multiphysics Simulation
READ NOW

PRESENTED BY V СОMSOL 


\title{
Anvil cell gasket design for high pressure nuclear magnetic resonance experiments beyond $30 \mathrm{GPa}$
}

\author{
Thomas Meier and Jürgen Haase \\ Faculty of Physics and Earth Sciences, University of Leipzig, Linnéstrasse 5, Leipzig 04103, Germany
}

(Received 6 November 2015; accepted 15 December 2015; published online 31 December 2015)

\begin{abstract}
Nuclear magnetic resonance (NMR) experiments are reported at up to $30.5 \mathrm{GPa}$ of pressure using radiofrequency (RF) micro-coils with anvil cell designs. These are the highest pressures ever reported with NMR, and are made possible through an improved gasket design based on nano-crystalline powders embedded in epoxy resin. Cubic boron-nitride (c-BN), corundum $\left(\alpha-\mathrm{Al}_{2} \mathrm{O}_{3}\right)$, or diamond based composites have been tested, also in NMR experiments. These composite gaskets lose about $1 / 2$ of their initial height up to $30.5 \mathrm{GPa}$, allowing for larger sample quantities and preventing damages to the RF micro-coils compared to precipitation hardened CuBe gaskets. It is shown that NMR shift and resolution are less affected by the composite gaskets as compared to the more magnetic $\mathrm{CuBe}$. The sensitivity can be as high as at normal pressure. The new, inexpensive, and simple to engineer gaskets are thus superior for NMR experiments at high pressures. (C) 2015 AIP Publishing LLC. [http://dx.doi.org/10.1063/1.4939057]
\end{abstract}

\section{INTRODUCTION}

High pressure physics evolved into one of the most intriguing branches of modern condensed matter physics, ${ }^{1}$ partly inspired by advances in anvil cells or multi-anvil press design, methods that allow for a significant change in the structure of solids. $^{2-4}$

Implementing NMR with high pressure apparatuses was believed to be extremely challenging, if not impossible, and only a few research groups succeeded with it. A first approach by Vaughan ${ }^{5}$ used a complex arrangement of split-pair coils wound around the flanks of both anvils in a Bridgman-type pressure cell. A similar set-up was used by Lee et al. ${ }^{6}$ in diamond anvil cells at pressures up to $6.8 \mathrm{GPa}$, which was also used by Bertani et al. ${ }^{7}$ Later, Lee et $a l .{ }^{8}$ introduced a significantly simplified radio frequency (RF) resonator that used a single-loop conductor. This design was used by other groups. ${ }^{9,10}$ Record pressures of up to $12.8 \mathrm{GPa}$ could be achieved using the gasket itself as a single-loop NMR coil by Pravica et al. ${ }^{11,12}$ These cell designs had the NMR coils placed far from the high pressure volume, and thus, diminished the filling factors and consequently the SNR (signal-to-noise ratio). Recently, we showed that high sensitivity NMR in diamond anvil cells is feasible by placing a multi-turn resonant RF micro-coil that encloses the sample tightly, directly into the pressure cavity. ${ }^{13,14}$ First experiments demonstrated the capability of this approach ${ }^{15,16}$ with retrofitted anvil cells originally used for de Haas-van Alphen type of measurements, with a micro-coil for low frequencies embedded in the pressure chamber. ${ }^{17}$ Subsequently, our group began developing its own NMR anvil cells, and in 2014, we showed that pressures up to $20.2 \mathrm{GPa}$ can be achieved at high sensitivity and good resolution. ${ }^{18-20}$ Simultaneously, Kitagawa et al. ${ }^{21,22}$ and Fujiwara et al. ${ }^{23}$ presented a similar approach reaching $10 \mathrm{GPa}^{24}$

Key components for all anvil cells, besides the anvils and their housing that stabilizes their position under load, are the gaskets that seal the sample chamber around the tips of the anvils. As external force is applied, the volume of the sample chamber that includes the sample decreases, resulting in pressures well into the range of several giga pascals (GPa). It is mostly the change in the gasket that accomplishes the task, and thus the performance of these pressure cells. The way the gasket changes under pressure can also be of great interest to measurement techniques that rely, e.g., on the shape of the sample chamber. This is true, in particular, for our high sensitivity NMR anvil cell designs. As the applied force changes the volume of the pressurized region, a decreasing gasket height can easily damage the micro-coil.

Over the last decade, several groups attempted to test new gasket designs based on so-called composite gaskets that are made from nano- or micrometer grain sized powder of a hard material like diamond, ${ }^{25,26} \alpha-\mathrm{Al}_{2} \mathrm{O}_{3}$ (corundum), ${ }^{27} \mathrm{c}-\mathrm{BN},{ }^{28}$ or boron carbide ${ }^{29}$ embedded in epoxy resin, replacing metallic gaskets at the regions of the highest pressures.

Typical arguments against the use of such insulating, composite materials, although chemically inert and extraordinarily hard, concern their low ductility and brittleness. Nonetheless, it was argued by Solli and Jeanloz ${ }^{29}$ that composite gaskets made from a very robust insulating material of very small grain size, much smaller than the size of the sample, and embedded in a rigid but relatively soft matrix of epoxy resin, will provide the necessary lateral stress on the sample cavity. Thus, it prevents a decrease in diameter, and with it the change in height of the gasket under pressure. If such a gasket is compressed, the soft epoxy matrix will be squeezed out of the regions of the highest stresses, allowing the remaining grains to densify and become more resistant to compression (the inter-locking of the grains increases, forming a hard supportive structure). Indeed, this approach yielded a much higher stability of the gasket, and ultimately higher attainable pressures in excess of $100 \mathrm{GPa}$.

As already mentioned, NMR experiments could greatly benefit from such a gasket design, since it prevents damage 
of the micro-coil in the high pressure region. And, indeed, this is our chief reason for the investigation of the use of such gasket designs within the presented work. However, given the importance of NMR in condensed matter physics and its potential for high pressure applications, ${ }^{15,16}$ non-metal gasket designs may also offer much greater flexibility for RF resonator designs in general, as opposed to metal ones that are impenetrable to RF. In addition, the new gaskets may also offer an alternative for reducing unwanted NMR resonances near the sample, e.g., for Cu NMR. Finally, as expected, the new gaskets are superior to most metallic ones when it comes to affecting NMR shifts and resolution.

Recently, we introduced a diamond/epoxy gasket with our high pressure cells and it enabled us to reach pressures of up to about $20 \mathrm{GPa}$ for the first time. ${ }^{20}$ Here, we discuss the performance of other composite gaskets and their use for NMR. They are based on different nano-powders of diamond, $\alpha-\mathrm{Al}_{2} \mathrm{O}_{3}$, and c-BN. We will use ruby powder to inspect pressure and pressure gradients within the cell, and perform ${ }^{27} \mathrm{Al} \mathrm{NMR}$ on the same ruby material for testing the NMR at up to $30.5 \mathrm{GPa}$ (under non-hydrostatic conditions). ${ }^{1} \mathrm{H}$ NMR at ambient pressure is used to determine the gaskets' influence on shifts and linewidths.

To the best of our knowledge, this manuscript represents the first application of composite gaskets to high pressure NMR experiments.

\section{EXPERIMENTAL}

The composite gaskets were manufactured as follows. The nano-powdered materials, diamond (grain size $\leq 50 \mathrm{~nm}$ from Schmitz-Metallographie $\mathrm{GmbH}$ ), $\alpha-\mathrm{Al}_{2} \mathrm{O}_{3}$ (grain size $\leq 200 \mathrm{~nm}$ from Nanostructured $\mathcal{F}$ Amorphous Materials, Inc.), and c-BN (grain size $\leq 1 \mu \mathrm{m}$, from LANDS Superabrasives $C o$.), were mixed with epoxy resin using a mortar and pestle in a volume ratio of about 5:1. Acetone was used to ensure a satisfactory mixing of the components. The mixture was dried in a furnace for $3 \mathrm{~h}$ at $80^{\circ} \mathrm{C}$. Small flakes were scraped off the resulting amorphous block using a ceramic knife.

Precipitation hardened $\mathrm{CuBe}(\mathrm{Cu} 98 \%$, Be 1.7\%-1.9\%, $\leq 0.5 \% \mathrm{Ni}$ and Co impurities, initial thickness of $500 \mu \mathrm{m}$, from Goodfellow Ltd.) was used to act as a girdle for the amorphous inner gasket under load, i.e., we removed the pre-indented region (about 20\%-40\% larger diameter than that of the culet) of a regular $\mathrm{CuBe}$ gasket, by wire erosion using an EDM, and filled it with the amorphous flakes of the composite materials. Thereafter, the assembly was again pre-indented to about twice the external force used to pre-indent the $\mathrm{CuBe}$ sheet in the first place. As a result, the amorphous flakes in the pre-indentation region transformed into a hard and robust disc that is confined by the outer $\mathrm{CuBe}$ gasket securing an outwards extrusion of the inner gasket under load. A non-magnetic tungsten carbide (WC) bit was used for drilling a hole for the sample chamber.

The channels (about $50 \mu \mathrm{m}$ deep) for incorporating the leads of the RF micro-coils were carved into the inner gasket using ceramic scalpels. We used RF micro-coils made from $18 \mu \mathrm{m}$ insulated copper wire from Polyfil $A G$ with an inner diameter of about $150 \mu \mathrm{m}-200 \mu \mathrm{m}$ of 7 turns. The coils

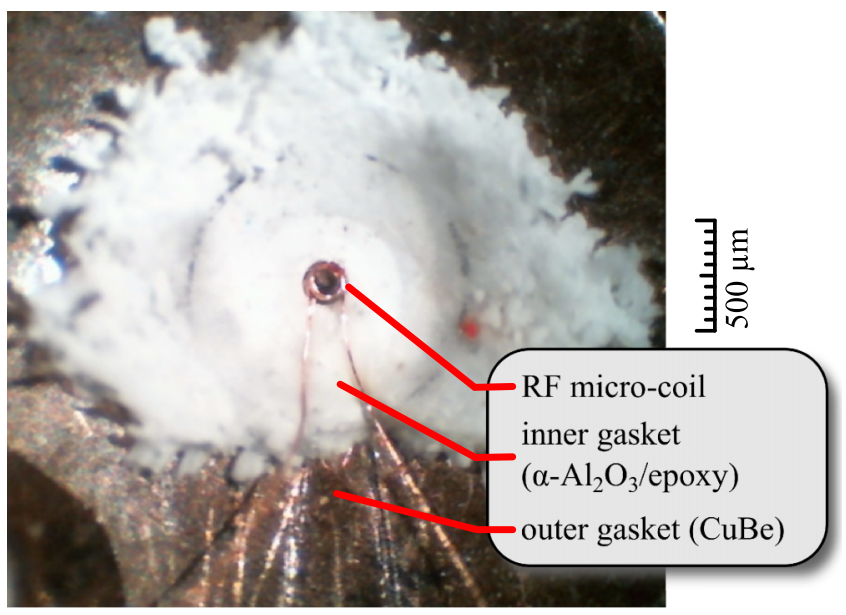

FIG. 1. Assembled composite gasket. The inner gasket made from $\alpha-\mathrm{Al}_{2} \mathrm{O}_{3}$ and epoxy is enclosed by the outer precipitation hardened $\mathrm{CuBe}$. The resonating RF micro-coil is placed in the center of the pre-indented flat area between the anvils' culet faces.

were placed inside the cylindrical $300 \mu \mathrm{m}$ sample hole. More details about the RF setup can be found in Ref. 20. The initial gasket heights were about $250 \mu \mathrm{m}$ in all pressure cells. Anvils with culet diameters of $600 \mu \mathrm{m}$, bevelled to $15^{\circ}$, were used to generate pressures well above $20 \mathrm{GPa}$. The demanding assembly of NMR resonators with CuBe gaskets in anvil cells was published elsewhere, ${ }^{19}$ and we refer to this publication for further discussion of that process.

The recovered thicknesses of the gaskets were measured with a micrometer, having an accuracy of $5 \mu \mathrm{m}$. Figure 1 shows the completely assembled composite gasket with the RF micro-coil. After implementation of the RF micro-coil, the whole sample chamber was filled with fine ruby powder, and the cell was pressurized with paraffin oil as pressure medium. The sample cavities were filled as described earlier. ${ }^{19}$

The pressure was determined with the pressure dependence of the ruby $R_{1}$ and $R_{2}$ photoluminescence lines. ${ }^{30} \mathrm{We}$ also swept the laser beam of the optical spectrometer in steps of $10 \mu \mathrm{m}$ across the pressurized region to determine the pressure gradient.

The cell was inserted into the home-built probes, as described elsewhere, ${ }^{18}$ and ${ }^{27} \mathrm{Al}$ NMR of the same ruby powder was performed using a Bruker HD III spectrometer at magnetic fields corresponding to proton resonance frequencies of $400 \mathrm{MHz}$ and $500 \mathrm{MHz}$. With separate experiments, ${ }^{1} \mathrm{H}$ NMR of tap water was used to measure the shift and linewidth at ambient pressure with various gasket materials.

\section{RESULTS}

First, we investigated the pressure dependence of the recovered gasket height for three different composites, cf. Fig. 2. We also include data from a $\mathrm{CuBe}$ gasket for comparison. Clearly, the composite gaskets retain a much higher thickness compared to the relatively soft metallic gaskets at all elevated pressures. We also observed a reduction in diameter of the sample chamber for both, composite and metallic gaskets after the releasing of the pressure for the open DACs. The diameter of the sample chamber of the composite 


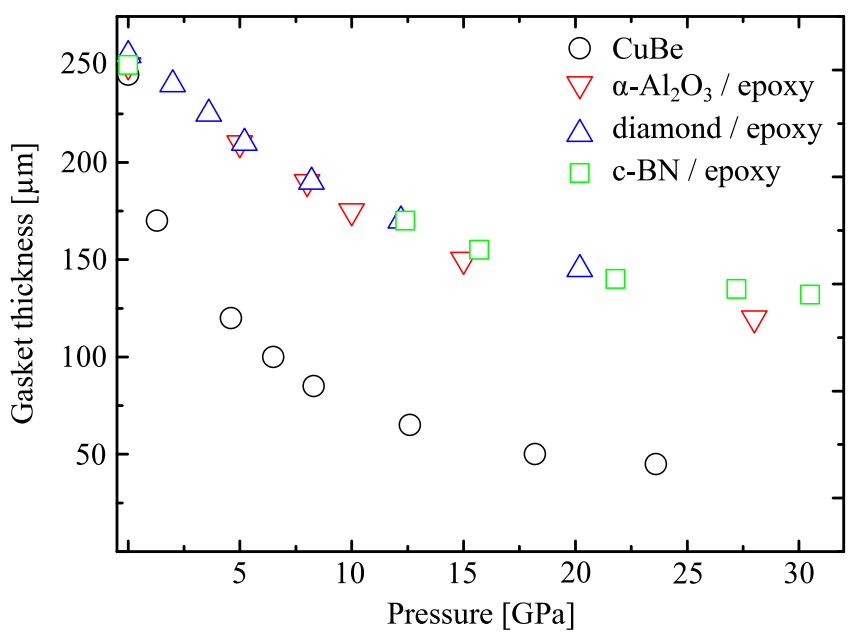

FIG. 2. Gasket thickness as a function of pressure.

gaskets had shrunk by about $40 \%$ of the original diameter for the cells that were at the highest pressure. The pure $\mathrm{CuBe}$ gasket was contracted even by $80 \%$ for the cell that was at $23 \mathrm{GPa}$ (while this was difficult to measure, it may indicate horizontal flowing of the gasket upon releasing of pressure).

We also recognize that the pressure performance is very similar for all three composites independent of the powder used. This adds flexibility to the actual NMR experiment where some materials should be avoided due to possible spurious background signals, except for ${ }^{1} \mathrm{H}$ NMR due to the extensive use of epoxy resin in the gasket material.

In addition to the improved pressure stability, the new gaskets are expected to have a smaller magnetic susceptibility, such that these gaskets are less perturbing for shift and linewidth of the enclosed sample material. To investigate this issue, we measured ${ }^{1} \mathrm{H}$ NMR of tap water in all three composite gaskets and a $\mathrm{CuBe}$ gasket. The data were compared to those obtained with a typical (much larger) NMR sample tube. The results are summarized in Table I. For the CuBe gasket, we found a shift of about $20 \mathrm{ppm}$, and a linewidth of about $4.5 \mathrm{ppm}$ (of course, the actual shifts and linewidths depend somewhat on the $\mathrm{CuBe}$ used, as well as the actual cell design). For the composite gaskets, we found much smaller shifts and linewidths, as expected. This shows that composite gaskets facilitate improved shift and linewidth measurements.

Next, we investigated the pressure distributions in the sample chamber, see Fig. 3. The loss of hydrostaticity is evident at pressures above $12.4 \mathrm{GPa}$. This is expected, as the approximate glass transition occurs at about this pressure for the paraffin oil used. The observed pressure gradients approached about $50 \mathrm{MPa} / \mu \mathrm{m}$. We observed no anomalies

TABLE I. Resonance shifts and linewidths from pressure cells using composite gaskets compared to metallic $\mathrm{CuBe}$, at $11.74 \mathrm{~T}$. $\Delta K$ denotes the shift in resonance frequency in the pressure cells relative to the same sample in a standard NMR set-up.

\begin{tabular}{lcccc}
\hline & CuBe & Diamond/epoxy & $\alpha$ - $\mathrm{Al}_{2} \mathrm{O}_{3} /$ epoxy & c-BN epoxy \\
\hline$\Delta K(\mathrm{ppm})$ & 20 & $\leq 2$ & $\leq 2$ & $\leq 1$ \\
FWHM $(\mathrm{ppm})$ & 4.5 & 1.6 & 1.8 & 1.5 \\
\hline \hline
\end{tabular}

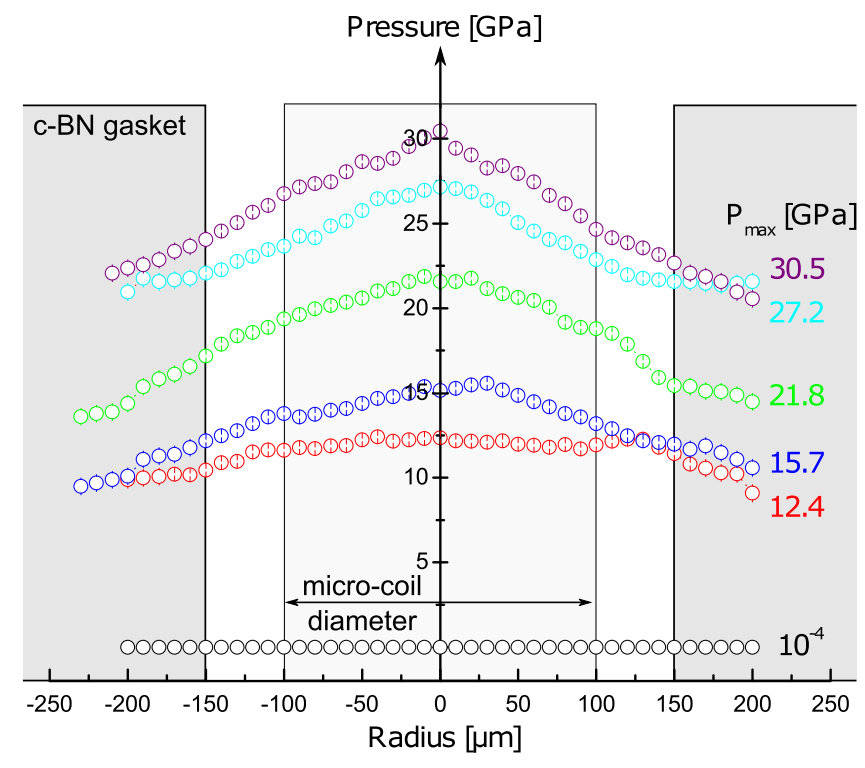

FIG. 3. Pressures as a function of radial distance from the center of the culet. Also shown is the approximate position of the c-BN/epoxy composite gasket, as well as the inner diameter of the RF micro-coil.

in the pressure distributions at maximal pressures of up to $30.5 \mathrm{GPa}$, and conclude that the composite gaskets work reasonably well up to that pressure regime.

Finally, we investigated the ${ }^{27} \mathrm{Al} \mathrm{NMR}$ of the ruby powder used for pressure determination. It is known that the crystal structure of corundum leads to a non-vanishing axially symmetric electric field gradient at the aluminium site with a quadrupole frequency of about $360 \mathrm{kHz} .{ }^{31}$ The ${ }^{27} \mathrm{Al} \mathrm{NMR}$ central transition is not affected by the quadrupole interaction in leading order (at our strong magnetic fields), while the four satellite transitions are shifted according to the orientation of the grains so that typical powder spectra appear, cf., Fig. 4. One can easily see the "horns" form the symmetrically distributed satellite pattern.

Under pressure, no structural phase transitions are known to occur below $80 \mathrm{GPa},{ }^{32}$ due to the very rigid coordination of

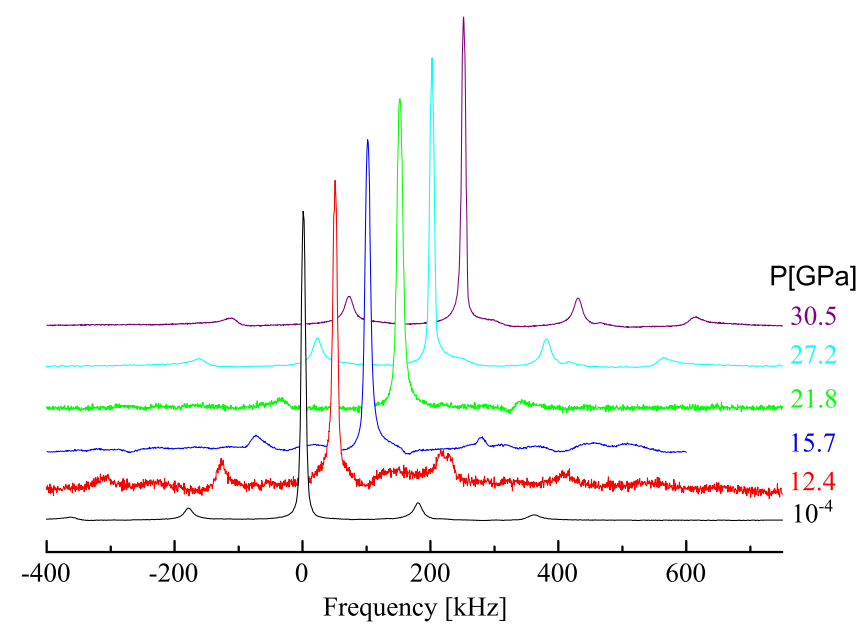

FIG. 4. ${ }^{27} \mathrm{Al}$ NMR Fourier transform spectra of $\mathrm{Cr} \mathrm{Al}_{2} \mathrm{O}_{3}$ at static pressures up to $30.5 \mathrm{GPa}$ (from FIDs excited with a non-selective $\pi / 2$-pulse of $500 \mathrm{~ns}$ at an rms pulse power of $2 \mathrm{~W}$ ). Spectra are shifted for better comparison and scaled to the height of the central line. The inner gasket was made from a c-BN/epoxy mixture. 
the oxygen octahedra surrounding the aluminum nuclei. This is indeed born out in our data shown in Fig. 4, since the quadrupole frequency, which is very sensitive to crystallographic changes, is basically independent on pressure.

Despite the large pressure gradient, the main features of the ruby spectra remained sharp, as well. This is also in agreement with the stability of the crystal structure. For each spectrum, about 9000 signals were averaged with a total measurement time of less than $10 \mathrm{~min}$ (the repetition time was $50 \mathrm{~ms}$ ). After opening the cells, the RF micro-coil in the cell working at $12.4 \mathrm{GPa}, 15.7 \mathrm{GPa}$, and $21.8 \mathrm{GPa}$ was found to be deformed, which might explain the lower signalto-noise with those spectra. Other than that, the signal-to-noise was rather independent on pressure. The signal-to-noise ratios were estimated as shown previously, ${ }^{19}$ and we find a value of about 2.4 at $30.5 \mathrm{GPa}$, which agrees with the estimated value of about 3.1. Therefore, we can conclude that the NMR micro-coil performs well even at the highest pressures.

\section{CONCLUSION}

We presented the first NMR experiments at pressures of up to $30.5 \mathrm{GPa}$, on ${ }^{27} \mathrm{Al} \mathrm{NMR}$ of ruby, which was also used to measure the pressure and its radial dependence in the anvil cell. In order to achieve these pressures with high sensitivity NMR using a RF micro-coil inside the pressurized region, we introduced composite gaskets made from a nano-powdered hard material and epoxy resin. The fabrication of the gaskets and implementation in the NMR pressure cells were described. As expected, we find these gaskets to be rather stable, with much smaller changes in height under pressure, as compared to gaskets made from precipitation hardened $\mathrm{CuBe}$. There appear to be no general sacrifices in terms of NMR sensitivity up to the highest pressures.

Since the composite gaskets are insulating, possible shortcircuiting of the leads of the RF coil is prevented. Moreover, we showed that these gaskets have a much smaller effect on shifts and linewidth, compared to $\mathrm{CuBe}$. We believe that composite gaskets will be essential to make NMR available at even much higher pressures.

\section{ACKNOWLEDGMENTS}

This research was funded by the International Research Training Group "Diffusion in Porous Materials" (DRK 1056) and the German Research Society (No. HA1893/12-1). We acknowledge the help of Tobias Herzig, Marko Staeter, and stimulating discussions with Sergej Medvedev, Mikhail Eremets, and Marianne Meier.

${ }^{1}$ A. P. Drozdov, M. I. Eremets, I. A. Trojan, V. Ksenofontov, and S. I. Shylin, Nature 525, 73 (2015).

${ }^{2}$ W. Grochala, R. Hoffmann, J. Feng, and N. W. Ashcroft, Angew. Chem., Int. Ed. 46, 3620 (2007).

${ }^{3}$ W. A. Bassett, High Pressure Res. 29, 163 (2009).

${ }^{4}$ R. J. Hemley, High Pressure Res. 30, 581 (2010).

${ }^{5}$ R. W. Vaughan, Rev. Sci. Instrum. 42, 626 (1971).

${ }^{6}$ S. H. Lee, K. Luszczynski, R. E. Norberg, and M. S. Conradi, Rev. Sci. Instrum. 58, 415 (1987).

${ }^{7}$ R. Bertani, M. Mali, J. Roos, and D. Brinkmann, Rev. Sci. Instrum. 63, 3303 (1992).

${ }^{8}$ S. H. Lee, M. S. Conradi, and R. E. Norberg, Rev. Sci. Instrum. 63, 3674 (1992).

${ }^{9}$ J. L. Yarger, R. A. Nieman, G. H. Wolf, and R. F. Marzke, J. Magn. Reson., Ser. A 114, 255 (1995).

${ }^{10}$ T. Okuchi, Phys. Earth Planet. Inter. 143, 611 (2004).

${ }^{11}$ M. G. Pravica and I. F. Silvera, Rev. Sci. Instrum. 69, 479 (1998)

${ }^{12}$ M. G. Pravica and I. F. Silvera, Phys. Rev. Lett. 81, 4180 (1998).

${ }^{13}$ J. Haase, S. K. Goh, T. Meissner, P. L. Alireza, and D. Rybicki, Rev. Sci. Instrum. 80, 073905/1 (2009).

${ }^{14}$ T. Meissner, S. K. Goh, J. Haase, B. Meier, D. Rybicki, and P. L. Alireza, J. Low Temp. Phys. 159, 284 (2010).

${ }^{15}$ T. Meissner, S. K. Goh, J. Haase, G. V. M. Williams, and P. B. Littlewood, Phys. Rev. B: Condens. Matter Mater. Phys. 83, 220517 (2011); e-print arXiv:1105.2009.

${ }^{16}$ T. Meissner, S. K. Goh, J. Haase, M. Richter, K. Koepernik, and H. Eschrig, J. Phys.: Condens. Matter 26, 015501 (2014).

${ }^{17}$ P. L. Alireza and S. R. Julian, Rev. Sci. Instrum. 74, 4728 (2003).

${ }^{18}$ T. Meier, T. Herzig, and J. Haase, Rev. Sci. Instrum. 85, 043903 (2014).

${ }^{19}$ T. Meier and J. Haase, J. Visualized Exp. 92, e52243 (2014).

${ }^{20}$ T. Meier, S. Reichardt, and J. Haase, J. Magn. Reson. 257, 39 (2015).

${ }^{21}$ K. Kitagawa, H. Gotou, T. Yagi, A. Yamada, T. Matsumoto, Y. Uwatoko, and M. Takigawa, J. Phys. Soc. Jpn. 79, 024001 (2010).

${ }^{22}$ K. Kitagawa, Y. Mezaki, K. Matsubayashi, Y. Uwatoko, and M. Takigawa, in Proceedings of the International Conference on Strongly Correlated Electron Systems (SCES2013) (J. Phys. Soc. Jpn., 2014), Vol. 015031, pp. $1-5$.

${ }^{23}$ K. Fujiwara, M. Iwata, Y. Okazaki, Y. Ikeda, S. Araki, T. C. Kobayashi, K. Murata, C. Geibel, and F. Steglich, J. Phys.: Conf. Ser. 391, 012012 (2012).

${ }^{24}$ M. Itoh, JPSJ News Comments 7, 03 (2010).

${ }^{25}$ R. Boehler, M. Ross, and D. Boercker, Phys. Rev. Lett. 78, 4589 (1997).

${ }^{26}$ D. E. Graf, R. L. Stillwell, K. M. Purcell, and S. W. Tozer, High Pressure Res. 31, 533 (2011).

${ }^{27}$ M. I. Eremets and I. A. Trojan, Nat. Mater. 10, 927 (2011).

${ }^{28}$ N. Funamori and T. Sato, Rev. Sci. Instrum. 79, 053903 (2008).

${ }^{29}$ D. Solli and R. Jeanloz, Rev. Sci. Instrum. 72, 2110 (2001).

${ }^{30}$ R. A. Forman, G. J. Piermarini, J. D. Barnett, and S. Block, Sci. (N. Y., NY) 176, 284 (1972).

${ }^{31}$ R. V. Pound, Phys. Rev. 79, 685 (1950).

${ }^{32}$ K. Syassen, High Pressure Res. 28, 75 (2008). 